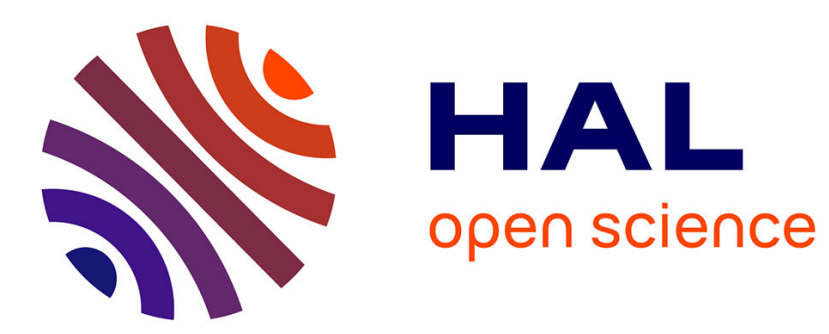

\title{
Experiments to Distribute and Parallelize Map Generalization Processes
}

Guillaume Touya, Justin Berli, Imran Lokhat, Nicolas Regnauld

\section{To cite this version:}

Guillaume Touya, Justin Berli, Imran Lokhat, Nicolas Regnauld. Experiments to Distribute and Parallelize Map Generalization Processes. Cartographic Journal, 2018, 54 (4), pp.322-332. 10.1080/00087041.2017.1413787 . hal-02155541

\section{HAL Id: hal-02155541 \\ https://hal.science/hal-02155541}

Submitted on 17 Jun 2019

HAL is a multi-disciplinary open access archive for the deposit and dissemination of scientific research documents, whether they are published or not. The documents may come from teaching and research institutions in France or abroad, or from public or private research centers.
L'archive ouverte pluridisciplinaire HAL, est destinée au dépôt et à la diffusion de documents scientifiques de niveau recherche, publiés ou non, émanant des établissements d'enseignement et de recherche français ou étrangers, des laboratoires publics ou privés. 


\title{
Experiments to Distribute and Parallelize Map Generalization
}

\section{Processes}

\author{
Guillaume Touya ${ }^{1}$, Justin Berli ${ }^{1}$, Imran Lokhat ${ }^{1}$, and Nicolas Regnauld ${ }^{2}$
}

1. Univ. Paris-Est, LASTIG COGIT, IGN, ENSG, F-94160 Saint-Mande, France

\section{1Spatial, Cambridge, United Kingdom}

\begin{abstract}
Automatic map generalization requires the use of computationally intensive processes often unable to deal with large datasets. Distributing the generalization process is the only way to make them scalable and usable in practice. But map generalization is a highly contextual process, and the surroundings of a generalized map feature needs to be known to generalize the feature, which is a problem as distribution might partition the dataset and parallelize the processing of each part. This paper proposes experiments to evaluate the past propositions to distribute map generalization, and to identify the main remaining issues. The past propositions to distribute map generalization are first discussed, and then the experiment hypotheses and apparatus are described. The experiments confirmed that regular partitioning was the quickest strategy, but also the less effective in taking context into account. The geographical partitioning, though less effective for now, is quite promising regarding the quality of the results as it better integrates the geographical context.
\end{abstract}

Keywords: Generalization; Partitioning; Parallelization

\section{Introduction}

Similarly as a text summary, map generalization seeks to abstract and simplify the geographic content of a map to display it at a smaller scale, where symbols are enlarged to ensure their legibility, and as a consequence, there is less space on the map to render map content without overlaps. When manually carried out, map generalization can be a very long task for a cartographer, so its automation has been studied by scholars and practitioners for years (Foerster, Stoter and Kraak 2010). Automatic map generalization processes are computationally intensive, and they are most of the time unable to deal with the size of real region wide or countrywide geographical datasets. In computer science, distributed computing is used to scale such complex processes by sharing the computation with multiple computers. Distributed computing often comes with parallel computing, which means that the process is cut into several sub-processes that are computed simultaneously on different computer cores. Distributing map generalization 
processes can help to both reduce the amount of data processed at once, and to reduce the computation time by parallelizing the processes. But map generalization is a wellknown holistic problem (Bundy, Jones and Furse 1995), as an automatic system needs to analyse the geographical context of any feature to decide what the best operation to apply is (Stoter et al. 2009). For instance, building generalization requires the recognition of implicit structures such as alignments (Basaraner and Selcuk 2008). If generalization is distributed, there is a major risk that quality might be damaged by hiding some parts of the geographical context. This problem is illustrated in Figure 1 with an example on road generalization, which needs some large context to decide what the important roads in an area are.

Some first proposals have been made to distribute generalization processes in the recent years (Chaudhry and Mackaness 2010), (Briat, Monnot and Punt 2011), (Thiemann et al. 2013), (Stanislawski, Falgout and Buttenfield 2015). But there is still no general guidelines on how to distribute a generalization process given the characteristics of the process and the dataset. That is why we propose to conduct some experiments to compare existing approaches, to analyse how much they can process large datasets without damaging the geographical context too much.

The second part of the paper describes the key problems of distributing generalization processes (partitioning, distributing context, parallelizing and reconciliation) and analyses the approaches of each problem in the literature. Then, the third part describes how we conducted our experiments. The fourth part shows and discusses the results obtained and the fifth part draws some conclusions and discusses further work.

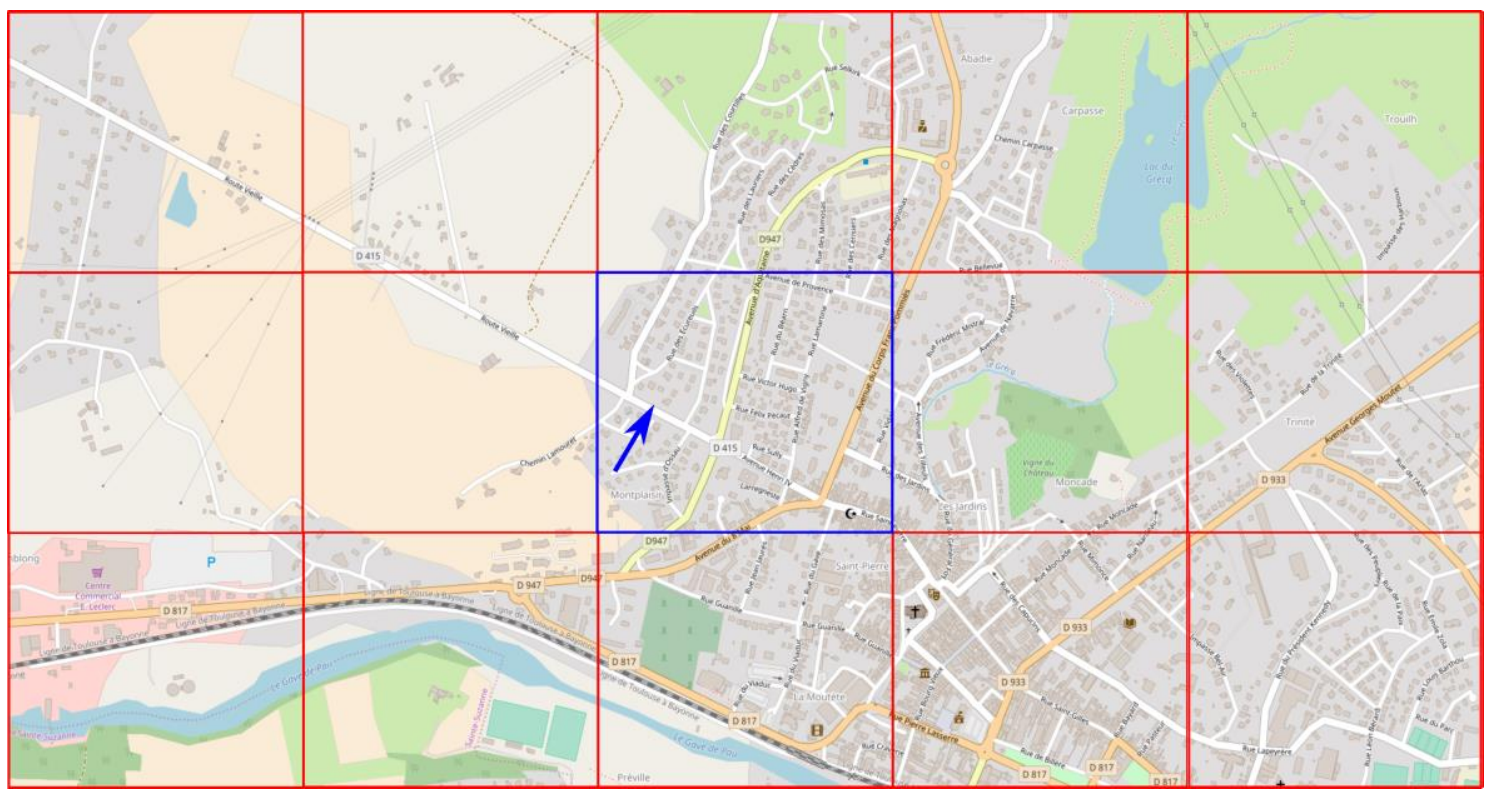

Figure 1. To generalize the roads of the center blue partition without surrounding context, it is not possible to consider the pointed road as the most important one, which it is considering this missing context (Source: @OpenStreetMap contributors). 


\section{Key Problems to Distribute Generalization Processes Introduction}

\subsection{Partitioning}

The first step to distribute a generalization process is to partition a large dataset into parts that are small enough to be manageable by the process. Two main approaches exist and have been tried in the literature: regular partitioning and geographical partitioning.

Regular partitioning includes two methods, the use of a regular (often rectangular) grid, or the use of a quad tree. Regular grids have been used for land use generalization by Thiemann et al. (2011), and for most generic processes in Briat, Monnot and Punt (2011) and Thiemann et al. (2013). The size of the grid is adjusted based on the amount of data that can be found in one cell of the grid. The quad tree is a smarter version of the regular grid with smaller cells used in more dense areas (Fig. 2), and Briat, Monnot and Punt (2011) showed that it can go faster than a simple regular grid.

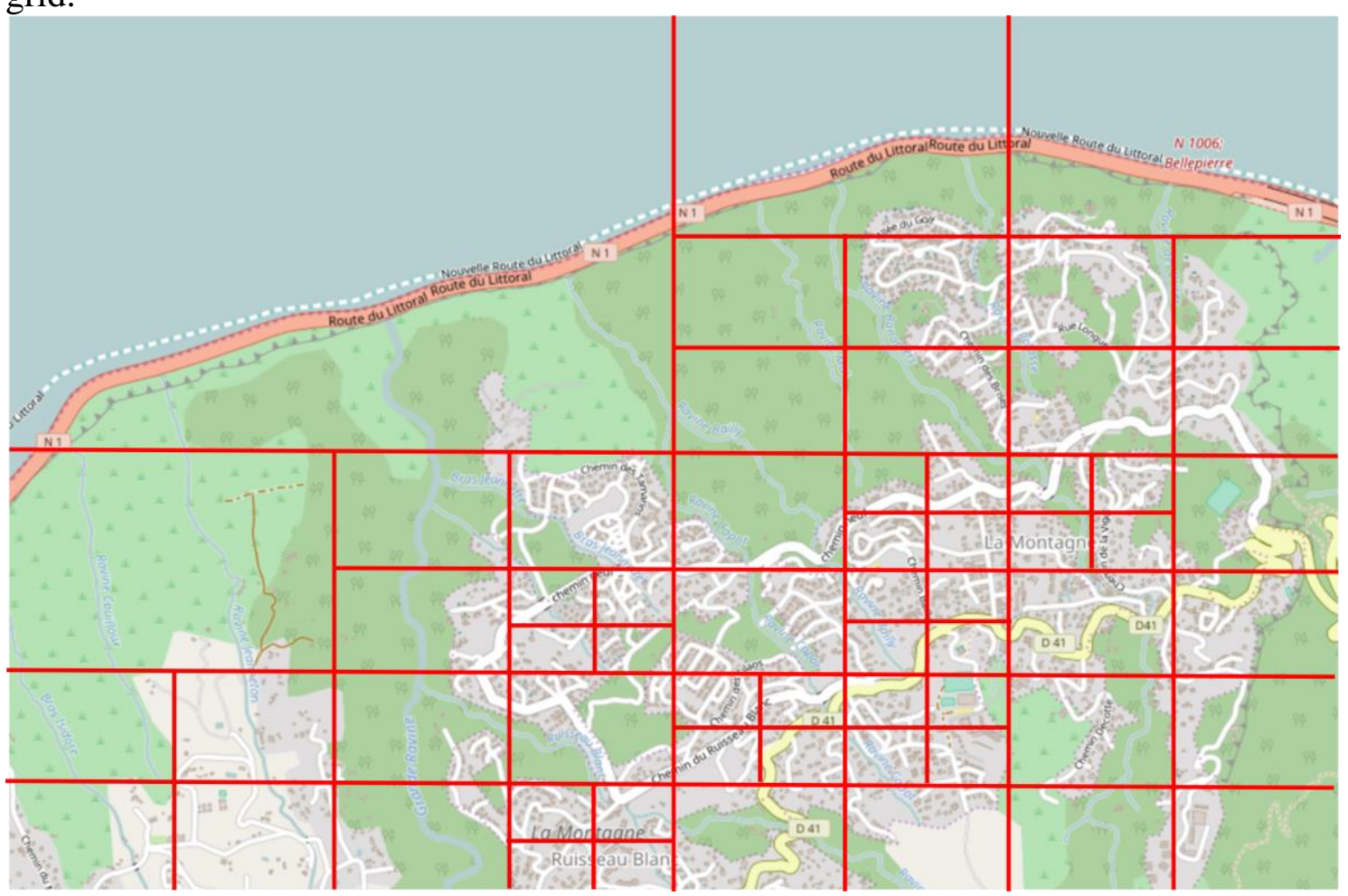

Figure 2. Illustration of the quad tree based partitioning: a cell of the map is divided in four equal cells as long as they are too many features in the cell (Source: @OpenStreetMap contributors).

An alternative strategy, described in (Chaudhry and Mackaness 2008), uses a geographical partitioning that would better capture the necessary geographical context while cutting data into small parts. A geographical partitioning can simply use features like administrative boundaries, as the US counties in (Briat, Monnot and Punt 2011). (Chaudhry and Mackaness 2010) proposed a combination of a regular grid and a geographical partitioning based on geomorphological regions, for DTM generalization. (Touya 2010) proposed a geographical partitioning based on main landscapes (urban, 
rurban, rural, mountain areas) with consideration for size (e.g. rural areas are cut by network elements and spaces without buildings to keep them small enough). (Thiemann et al. 2013) proposes to cluster data based on proximity (Anders 2003) to create meaningful partitions.

\subsection{Distribution of Geographical Context}

Once a partitioning technique has been chosen, it is necessary to add a mechanism to restore some kind of geographical context, at least for the features that are located near the boundary of a partition cell. The main proposition in the literature is to provide a buffer area around the partition where data is added for context, but not generalized by the process that handles the partition cell (Briat, Monnot and Punt 2011), (Thiemann et al. 2011). One difficulty here is to find the buffer size that gives enough context without making the partition cell too large to be processed. (Thiemann et al. 2013) proposes a classification of the generalization operations that requires a large context, and the ones that can be triggered without any regard for the context. For instance, there is no need to look at the neighbours to simplify the shape of a building polygon, but network selection requires a large context (Fig. 1).

\subsection{Parallelization}

Different methods exist to parallelize the processes, even if the differences are more technical than conceptual. Parallelizing a process means that several nodes, that is cells of the partition, are processed in parallel by different computer cores, that can be in a same machine (Briat, Monnot and Punt 2011), or not, and even in a machine cluster (Stanislawski, Falgout and Buttenfield 2015). There are some interesting details to tackle: for instance if the system allows multiple or single reads or writes on the dataset, or if some asynchronous mechanisms are used for adjacent partition cells (Briat, Monnot and Punt 2011).

If the Map/Reduce framework is used for parallelization (Thiemann et al. 2013), there are also implications for the way processes are implemented, and reimplementations might be required.

\subsection{Reconciliation}

The final issue is the reconciliation of the data processed in parallel, i.e. decide what to do if a feature has been included in several partition cells. (Thiemann et al. 2013) calls this step composition. Briat, Monnot and Punt (2011) use an attribute field on features that says if it has been processed, and only the first processed partition is able to write the result on the feature at the reconciliation step. Thiemann et al. (2011) cut the features at the limits of the partition and reconciliate by merging the cut parcels once generalized. However, cutting features is dangerous as shown in (Chaudhry and Mackaness 2008) with the example of Douglas \& Peucker algorithm (Douglas and Peucker 1973) that fixes the initial/final vertices of the simplified lines, so more line cuts means more fixed points and less quality in generalization. 
Thiemann et al. (2013) discusses three methods for reconciliation: selection (objects on the boundary of partition can be selected in only one partition cell), cut and merge, and match and adjust (objects are generalized in parallel and their new representation is matched and adjusted).

\section{Description of the Experiments}

\subsection{Hypotheses}

Large datasets can cause two problems when generalized: too long processes and crashes because of the amount of data to process. The distribution techniques have to find the balance between computation time, the maximum amount of data processed at once, and the cartographic quality of the generalized map. We made four hypotheses about this balance between the three objectives of distribution:

- Optimising computation time or the amount of processed data does not optimise cartographic quality (H1).

- Regular partitioning is a better choice for non-contextual processes (H2).

- Geographical partitioning is better for contextual processes (H3).

- There is no generic best distribution method; it may depend on data types and generalization processes $(\mathbf{H 4})$.

- Distribution is not really platform dependent (H5).

\subsection{Case Study}

We used a large dataset to experiment different distribution strategies, extracted from IGN (the French national mapping agency) topographic database that contains geographical data with a 1 meter geometric resolution. Data from Reunion Island was used as input data in our experiments. Reunion Island located in the Indian Ocean has been chosen mainly because of the variety of geographical features that it presents (dense cities, rural areas, dense hydrographic network, etc.), on an area large enough (2 $512 \mathrm{~km}^{2}$ ) to cause issues for many non-distributed processes. We processed buildings, roads, rivers and coastlines from this dataset.

The major part of the experiments was conducted on CartAGen (Renard, Gaffuri and Duchêne 2010), that is the module dedicated to generalization in the open source GeOxygene Java platform (Bucher et al. 2012). For the sake of simplicity, we distributed the processes of CartAGen using the Java Parallel Processing Framework (JPPF), which only requires minimum refactoring of the code to distribute Java software. The cluster used to process the data is composed of 5 standard desktop computers, their computing power being uneven. Each computer has 4 cores, so is able to run four processes in parallel. In order to test (H5), we also carried out experiments with the commercial platform 1Generalize from 1Spatial, which uses its own distributed system based on Oracle Weblogic Server.

For the remainder of the paper, we call a node a unitary element of the architecture able to run one process. In our architecture a node is one core of one of the available computers. We call a job the processing of a partition cell by one node. 
For the purpose of the tests, two algorithms were chosen: the polyline simplification algorithm from (Visvalingam and Whyatt, 1993), (Visvalingam and Whelan, 2016) and the building squaring algorithm currently developed at the IGN (Lokhat and Touya, 2016). The simplification algorithm is contextual because it was enhanced to avoid topological errors with the other lines of the dataset. A high parameter value (2000 square meters) was chosen for the effective areas of the polyline simplification algorithm to highlight possible topological inconsistencies. The squaring algorithm is not contextual, each building being processed without any consideration for its neighbours. It will be used to test (H2).

\subsection{Description of the Experiments}

Each distribution experiment was carried out with three different configurations of available nodes to distribute the process:

- A single computer with four nodes (a total of 4 nodes).

- Five computers, with a single node each (a total of 5 nodes).

- Five computers with four nodes each (a total of 20 nodes).

The limitation to three configurations guarantees a small amount of instances of the experiments, but these particular configurations offer a different number of nodes, to verify that more nodes lead to faster processing times. These configurations also allow the comparison between nodes on the same computer and nodes on different computers for a similar number of nodes.

\subsubsection{Experiments with Regular Partitions}

We first carried out experiments with the simplest partitioning method, the rectangular grid. Two types of entities are treated using this partitioning solution, polylines (streams, roads and coastlines) and polygons (buildings). Regarding lines, the regular rectangular grid is implemented following two processing methods to handle context (Fig. 3a and b): one creating a buffer around each cell to provide a context for the simplification of polylines; the other cutting each lines according to the processed cell boundaries.

In the first method, it has been decided to use a buffer that includes each of the eight surrounding cells (Fig. 3a). The lines that intersect the buffer are loaded into memory, then their centers (located on the line) are calculated. If that point is inside the cell, the line will be processed, if not, it will be used as a context for the simplification algorithm in order to check any potential topology error caused by line intersections. If the center point of a line is located on the boundary of the cell, its identifier is stored and it will be treated afterwards, during the reconciliation stage. However, the error checking is done with the initial version of the lines, not the simplified ones, so intersections still might occur with the simplified lines. To avoid this problem, some asynchronous distribution should be used. 


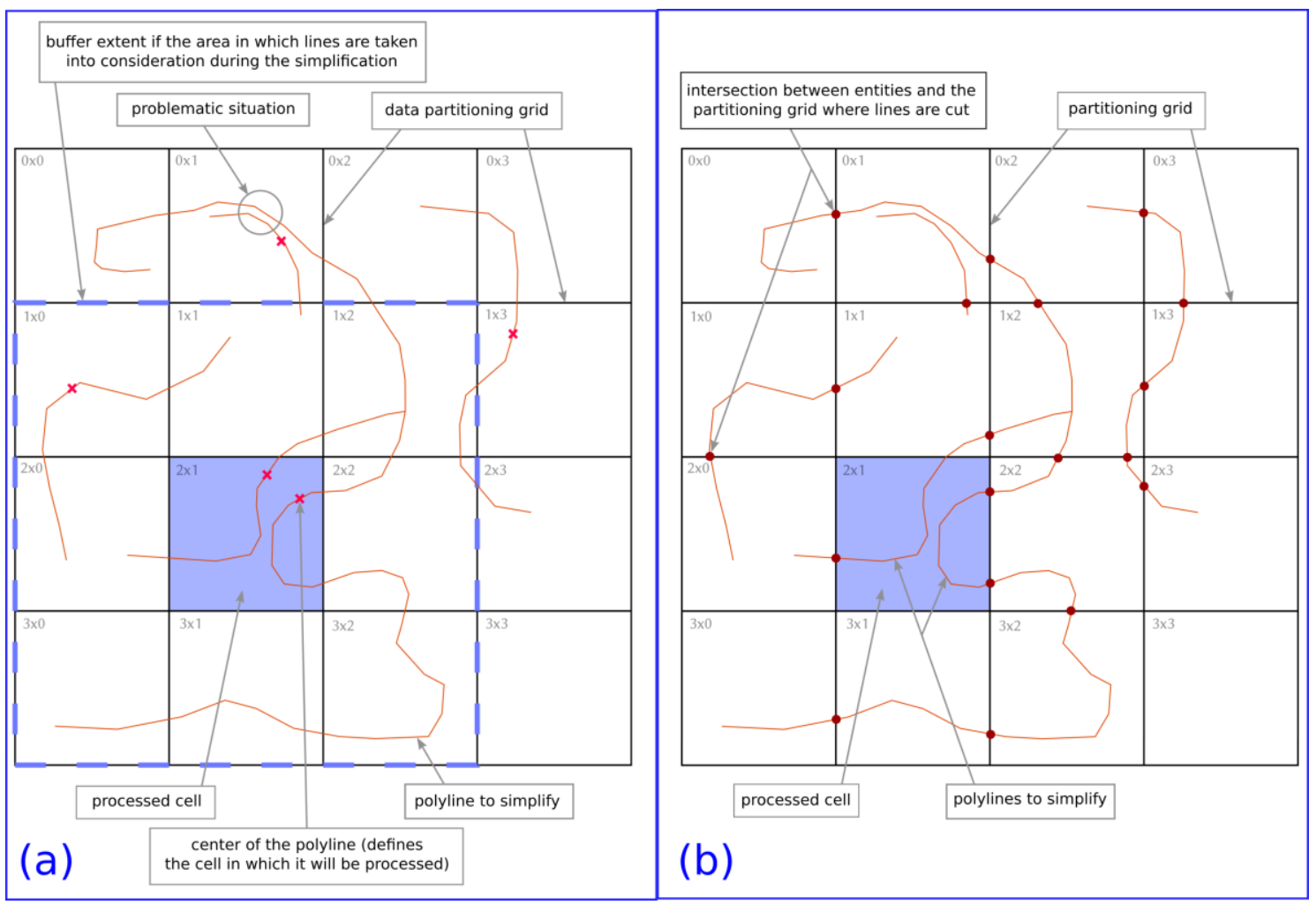

Figure 3. Two strategies to handle context in a regular grid partition: (a) a buffer area around each cell; (b) cutting features at the edge of partition cells.

In the second method (Fig. 3b), all the new lines created during the splitting phase must keep an attribute indicating the initial line id. This allows the reconciliation stage to recreate initial lines by aggregating all the sections that have the same attribute.

Processing buildings using the regular grid is the simplest method to set up as the centroid of each entity defines in which cell it will be processed (Fig. 4). When a centroid is located on top of a cell's boundary, which does happen when processing very large datasets (a dozen instances in our building dataset), its identifier is stored and the building is processed at the end, during the reconciliation stage.

We also carried out experiments with a quad tree regular partition, as it allows to keep a similar number of features in each cell, by dividing cells where the feature density is high. But the results highlighted a performance issue in our implementation of the quad tree, making the quad tree less effective than the regular grid, which is not consistent with past research (Briat, Monnot and Punt 2011). That is why we do not present any result with the quad tree in this paper. 


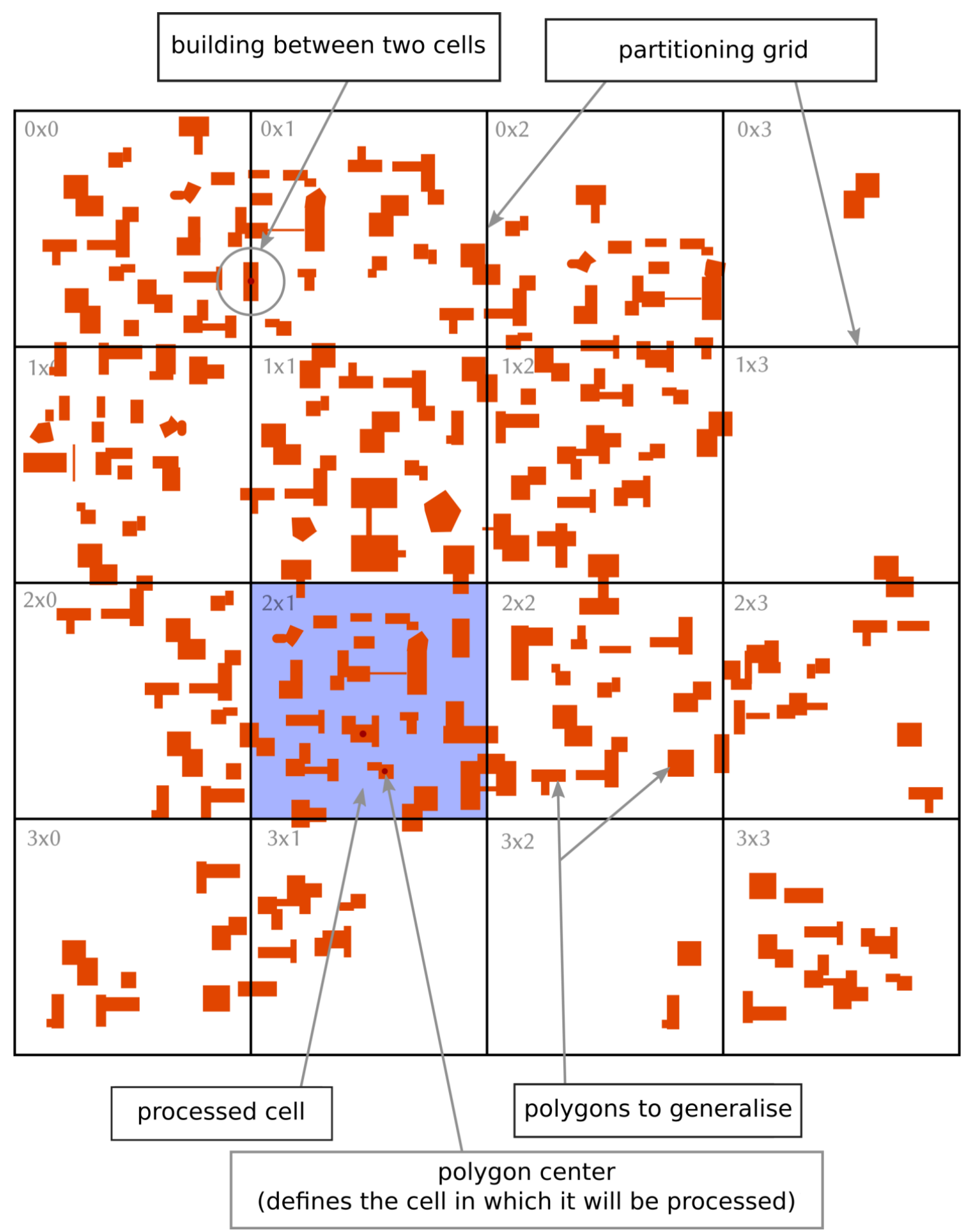

Figure 4. Regular grid applied to building squaring: the building centroid is used to assign a building to a cell; buildings with the centroid on a partition edge are assigned to a final reconciliation job.

\subsubsection{Experiments with Geographical Partitions}

The geographical partitioning methods use different types of geographical features to make small regions. In order to test $(\mathrm{H} 4)$ that makes the assumption that geographical 
partitioning are adapted to some features types but not to all, it has been decided to work on three feature types to offer a variety of results for them to be compared: roads, rivers, coastlines. As all three types of features are linear, the same simplification algorithm is applied (Visvalingam-Whyatt). Three types of geographical partitioning are also tested: administrative boundaries of cities that is supposed to be quite neutral for all three feature types, watershed extents that are supposed to be adapted to river lines, and divisions based on the road network that are supposed to be adapted to road lines.

The partitioning according to the administrative boundaries as well as to the watershed extents is achieved following the same workflow as for the segmentation method. Lines are loaded, split, processed and reconciliated the same way, only the mask changes, from a regular grid it becomes a complex polygon geometry (which will cause some issues discussed in the last section).

The partitioning according to the road network was performed on the 1 Generalize platform. It uses areas enclosed by road sections to create polygons which will be used as partitions. All small partitions formed inside the network are aggregated according to two main parameters: the maximum number of partitions to be merged and the maximum area allowed for a single partition. This method produces partitions which have various sizes but similar amount of data, producing smaller partitions in dense urban areas and larger ones in rural areas. This ensures that the processing time is fairly homogeneous across the partitions, for a more efficient load balancing across the grid of processing nodes. Using roads to partition space is a classical method in generalization, as roads often delimit the spatial context necessary to generalize features (Ruas and Plazanet 1996), (Burghardt and Neun 2006), (Touya 2010), (Briat, Monnot and Punt 2011). The processing then follows the same workflow as the previous method, cutting every line according to the boundaries of the partitions. Choosing the roads as partitioning features also helps keeping the number of split features down, and therefore limits the need for adding fixed points which are not ideal for the quality of the result.

\subsection{Operational Limitations}

To properly understand the results presented in the following, it is first mandatory to consider the material limitations as well as the other issues inherent to the method deployed. One has to take into consideration the memory limits of every node which happen when the partitions sent to the cluster hold too many entities. This phenomenon is observable when the regular grid used contains a small number of cells (Fig. 5) or when the extent of the geographical object - such as the administrative areas or the watersheds - is too large (Fig. 8); those can induce the presence of a large number of lines or buildings to be treated at once by a node. With more memory on each node, the balance between speed and cell size could be different.

Another limitation lies in the use of too many partitions that can lead to a significant growth in processing time. Indeed, in that case, the implemented distribution framework faces network congestions leading to failures with some jobs. This is not a major problem because it would be avoided by using a real grid architecture, and a more sophisticated distribution framework than JPPF. JPPF is a black box that gives minimal control on the parallelization step. For instance, using the Spark framework, already used for the analysis of large Lidar point clouds (Brédif, Vallet and Ferrand 2015), enables parallel processes that share input or output datasets, which can be useful to deal with the spatial context of a given generalization algorithm. Finally, the hardware differences inside the cluster must be considered, one of the processor being weaker 
than the other four, the randomness of the node assignation can lead to different results with the use of the same architecture and the same number of partitions. We believe that this is not a standard configuration, and it may cause biases preventing the comparisons between two strategies. So to overcome this limitation, we carried out each experiment several times and picked only the quickest results, i.e. the ones where the weak node of the grid did not handicap too much the process.

\section{Results and Discussion}

This section presents and discusses the results obtained with regular grids and geographical partitioning in the experiments described above.

\subsection{Regular Grid}

Concerning the results obtained with the regular rectangular grid, the first thing to notice is the overall lower speed of the method that cuts every line according to the cells boundaries. The duration is around 2 to 3 times higher when using the contextual method (Fig. 5 and 6). This is due to the reconciliation step that is not necessary with the contextual method.

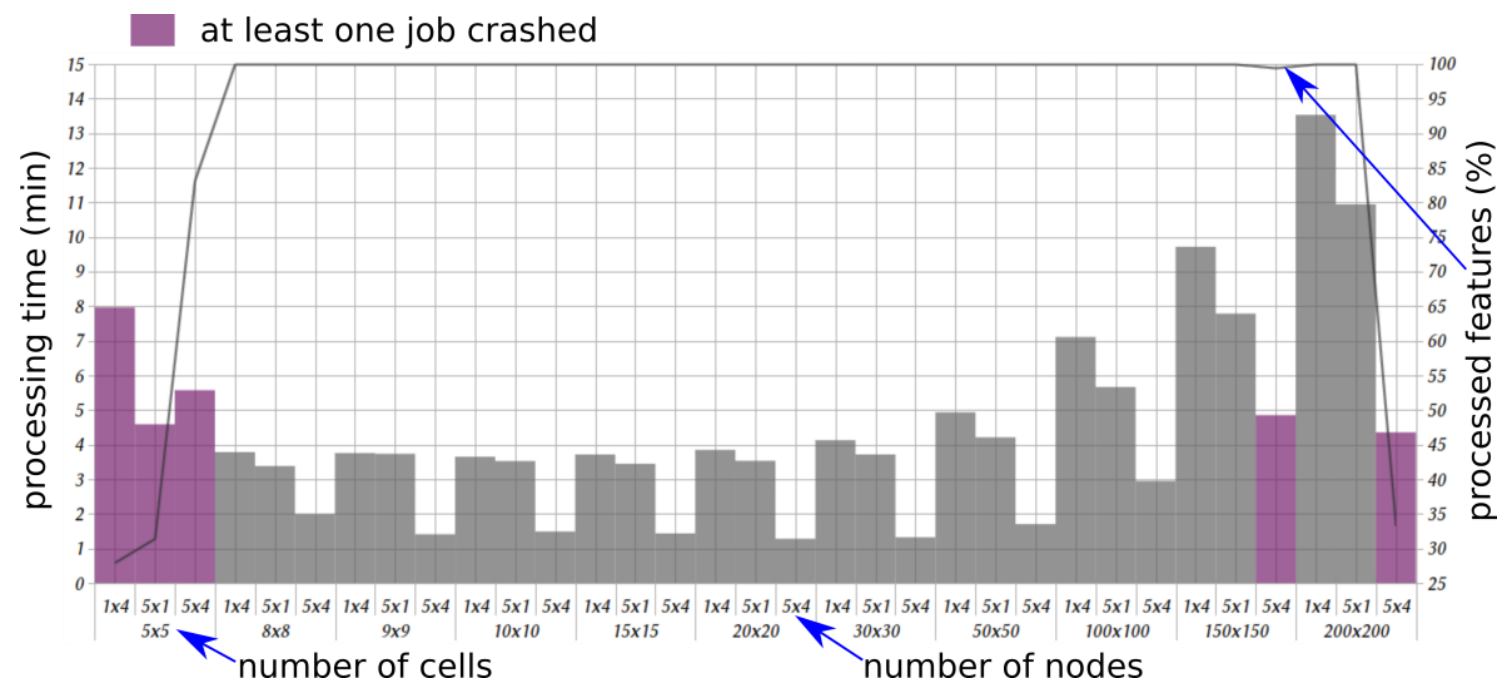

Figure 5. Results of the experiments with a regular grid for the generalization of rivers with the Visvalingam-Whyatt algorithm. 


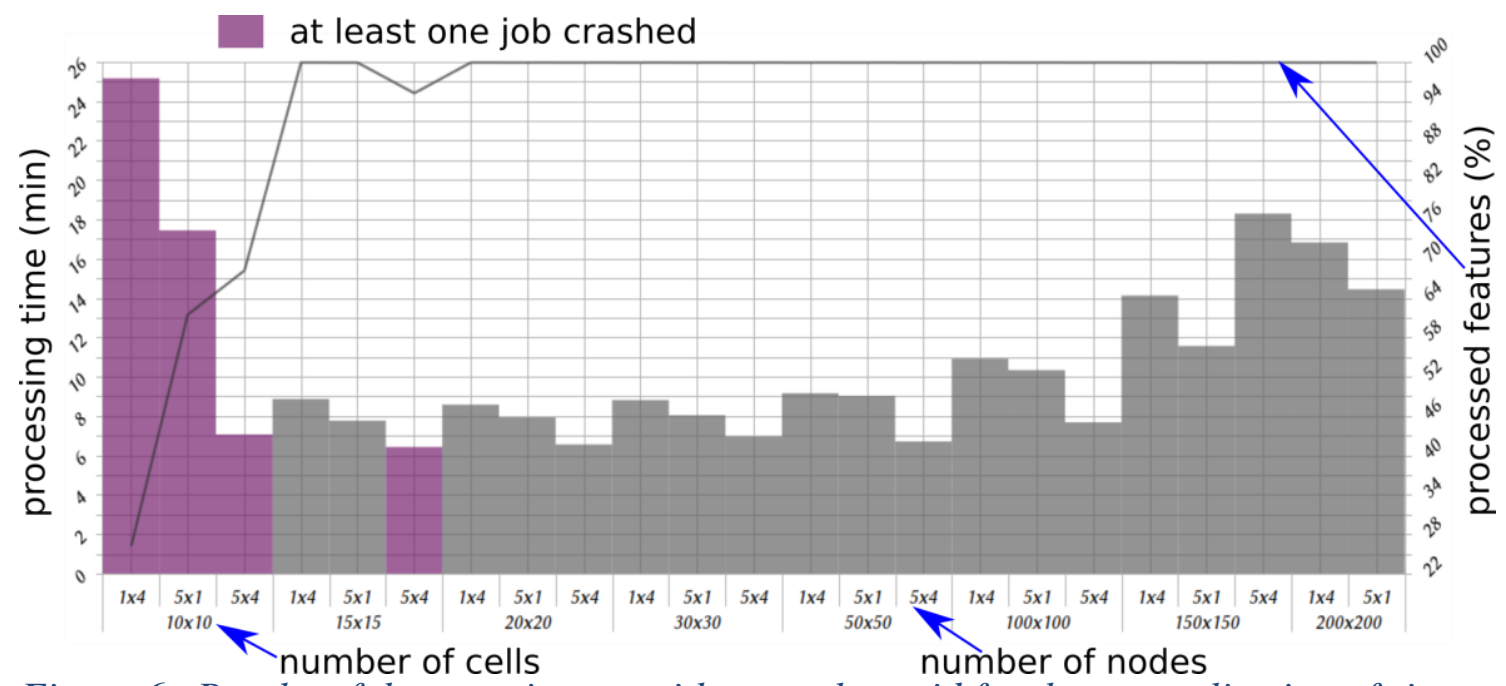

Figure 6. Results of the experiments with a regular grid for the generalization of rivers with the Visvalingam-Whyatt algorithm.

The difference increases along with the number of nodes used in the cluster. That being mentioned, one must consider the quality of the data obtained as well. The results (showed in Fig. 5 and 6) show that there is a minimum processing time around $20 \times 20$ grids, which corresponds to $2.5 \times 2.5 \mathrm{~km}$ cells on Reunion Island. We can also see that the architecture with five computers and four nodes by computer is the best one as predicted. The fall in the number of generalized features in the case with 200x200 cells and a $5 \times 4$ architecture illustrates the limitations of our framework, the high number of nodes to manage leading to network congestion and job failures.

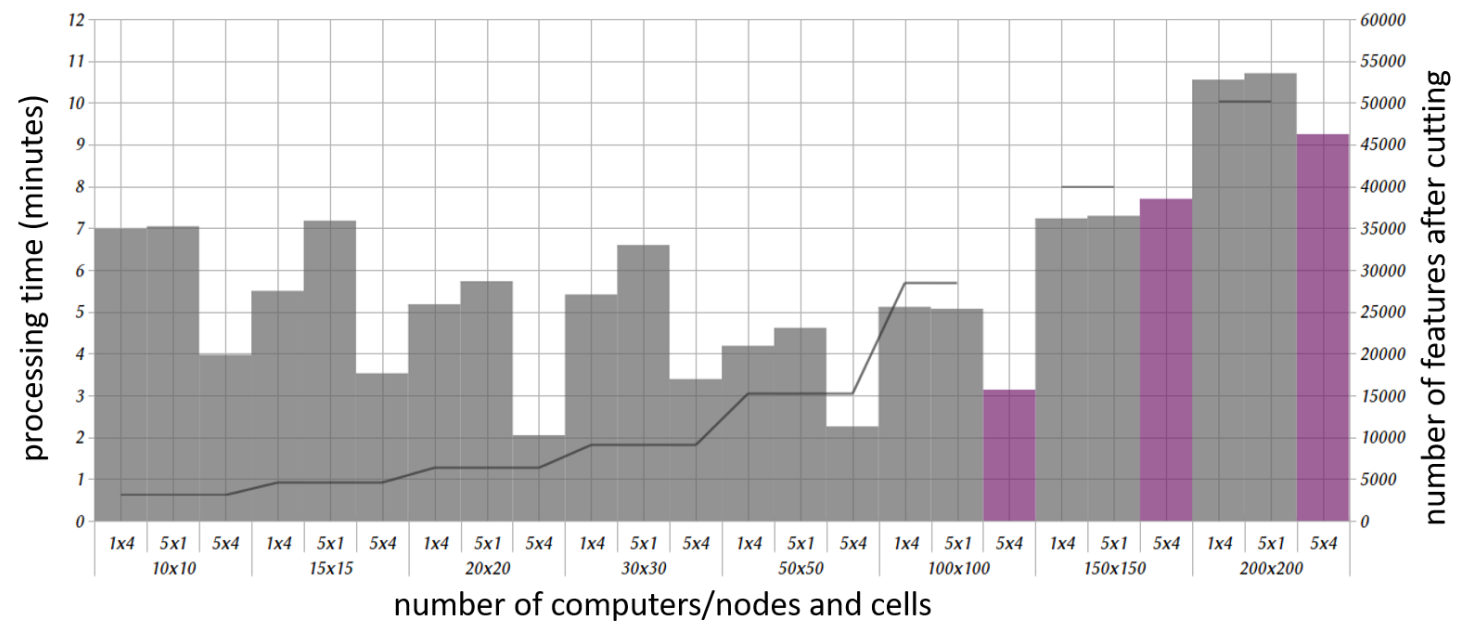

Figure 7. Results of the experiments with a regular grid for the generalization of roads with the Visvalingam-Whyatt algorithm. The right vertical axis corresponds to the black line, and gives the number of features after cutting: the more features are cut, the worse generalization is.

The method that splits the lines generates fixed points at every partition boundary, as extremities are kept in place during the simplification process. The contextual method produces data showing no trace of the partitioning stage as the algorithm considers the whole line while simplifying. Fig. 7 shows that the optimal configuration of nodes and partitions is similar, but large partitions are preferred as they minimise the number of cut features. Fig. 8 shows an example where cutting lines leads to a result very different from the buffer method output: the line is less simplified. The 
number of lines to reunite during the reconciliation stage then constitutes an indicator of the quality of the obtained data; the more lines needing to be aggregated, the worse the quality become. This proves that (H1) is true: the method that optimizes processing time and memory load does not provide the best cartographic results.

In the case of the building squaring, a non-contextual algorithm, the use of a regular rectangular grid provides generalized buildings very quickly, and way more quickly than the geographic partitioning. This result shows that $(\mathrm{H} 2)$ is true, there is no need for geographical partitioning when the generalization operation is not contextual. However, that method is quite unstable and sensitive to density differences: using too many cells gives worse results, but with fewer cells, the ones with more building density crash because of the memory load. As shown by Briat, Monnot and Punt (2011) a quad tree based method that makes more cells in dense areas would be the optimal solution.

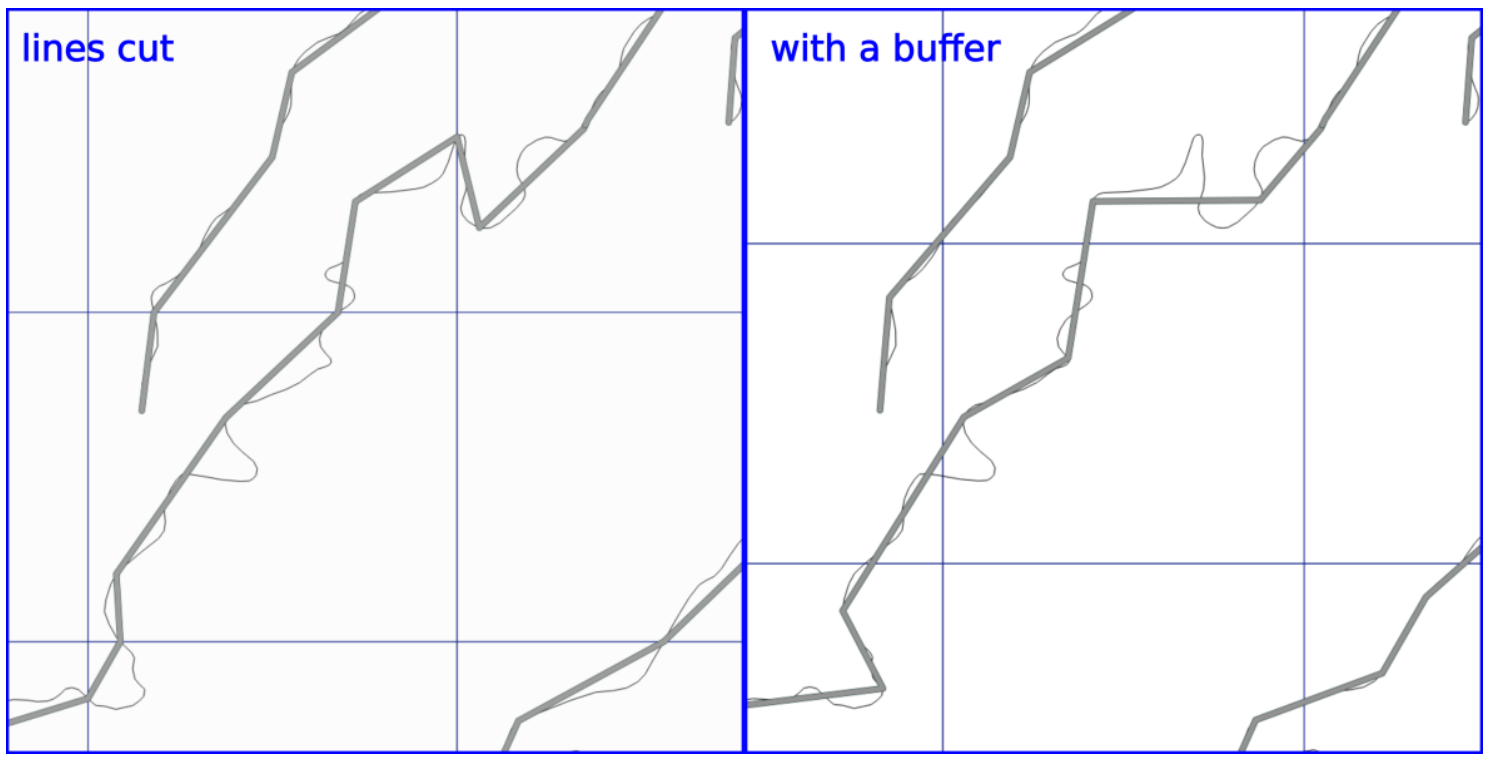

Figure 8. Differences in the cartographic output for Visvalingam-Whyatt simplification of rivers when using a buffer for context, or when cutting lines.

\subsection{Geographical Partitioning}

First, both the use of administrative boundaries and watersheds extent imply a significant limitation. The complexity of the geometries used to split lines is too important for the spatial query to be time-efficient. The difference observable with the use of a similar number of rectangular cells - which are simple-shaped polygons - is really noticeable and makes geographical partitioning ineffective for now. Another limitation is that the cells of the geographical partitions we used were too big compared to the optimal cell size found with the previous experiments. Further experiments are clearly necessary to overcome these limitations that prevent us from asserting that $(\mathrm{H} 3)$ is true, i.e. geographical partitioning is better for contextual generalization processes.

Nevertheless, our results give us hints on (H3). For instance, the use of watersheds as masks to split water streams, could be, if optimized, a way to enhance time-efficiency while preserving the quality of the data. Indeed, streams only cross watersheds boundaries at one point, the outlet, and the results have a much better quality (Fig. 9). Partitioning the dataset according to zones derived from the road network allows the same kind of principle as no road crosses another. The limitation for 
now lies in the fact that the whole network needs to be simplified before the creation of the actual partitions. This can induce some issues, particularly if the nodes composing the cluster have limited cache memory. The results obtained using this method show that the partitioning does not reflect on the quality of the simplified roads.

More generally, it might be difficult to assert that (H3) is true or false for all contextual processes. For instance, Fig. 8 shows that river streams simplification mostly requires the minimisation of intersections between the partition cells and the streams, while building typification does require a view of the buildings neighbourhoods to identify and preserve patterns, which cannot be guaranteed by a regular grid.

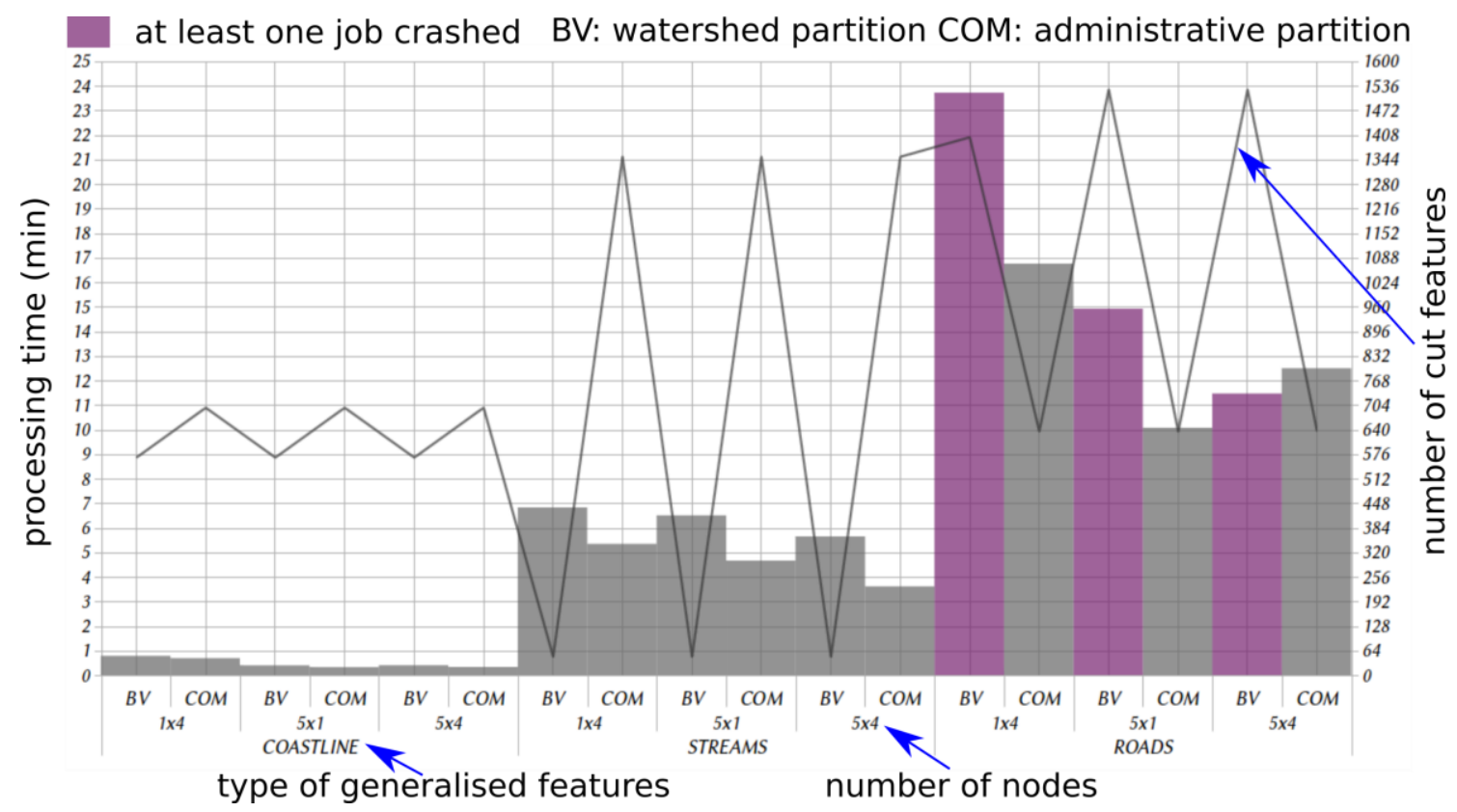

Figure 9. Synthesis of the results for the simplification of lines using geographical partitioning based on watershed extents and administrative limits.

These first results also suggest that $(\mathrm{H} 4)$ might be true: the best distribution strategy depends on the feature types processed, and on the fact that the generalization process itself is more or less contextual. Apart from the current limitations we believe that the watershed based partitioning is the best when processing only rivers with a contextual algorithm such as simplification but also selection that is often carried out by watershed analysis (Stanislawski, Falgout and Buttenfield 2015).

The results obtained on the 1 Generalize platform (Fig. 10) with the road network based partitioning show similar patterns between the number/size of cells and the processing time, with slight differences that might be due to differences in algorithm implementation and in the distribution architecture. One obvious difference lies in the fact that the processing nodes load the data in a local cache. This slows down the process, but removes the risk of failure due to lack of memory if the node is given a large area to process. However, we think that the patterns are similar enough to consider that (H5) is probably true: the platform differences are not significant compared to the differences due to partitioning and context handle methods.

In addition to the platform comparison, this experiment with 1 Generalize confirms both (H4) and (H5) as the road partitioning is suitable for buildings and coastlines, but clearly not for rivers, even when the partition cells are large. 


\begin{tabular}{|c|c|c|c|c|c|c|c|}
\hline \multirow{3}{*}{$\begin{array}{l}\text { Number } \\
\text { of } \\
\text { partitions }\end{array}$} & \multirow{2}{*}{\multicolumn{3}{|c|}{$\begin{array}{l}\text { Processing time } \\
\text { (HH:MM:SS) }\end{array}$}} & \multicolumn{3}{|c|}{$\begin{array}{l}\text { Number of features crossing a } \\
\text { partition cell }\end{array}$} & \multirow{3}{*}{$\begin{array}{l}\text { Number of } \\
\text { jobs with } \\
\text { errors }\end{array}$} \\
\hline & & & & \multirow[b]{2}{*}{ Buildings } & \multirow[b]{2}{*}{ Rivers } & \multirow[b]{2}{*}{ Coastlines } & \\
\hline & $\begin{array}{l}\text { Partitioning } \\
\text { step }\end{array}$ & $\begin{array}{c}\text { Genera } \\
\text { lisation } \\
\text { step }\end{array}$ & Total & & & & \\
\hline 0 & & 1:09:55 & 1:09:55 & & & & 0 \\
\hline 9 & $0: 13: 10$ & 0:32:07 & $0: 45: 17$ & 0 & 1,039 & 4 & 0 \\
\hline 11 & $0: 13: 02$ & $0: 31: 16$ & $0: 44: 18$ & 0 & 1,135 & 4 & 0 \\
\hline 14 & $0: 12: 27$ & $0: 23: 40$ & $0: 36: 07$ & 1 & 1,238 & 4 & 0 \\
\hline 18 & $0: 13: 56$ & $0: 27: 31$ & $0: 41: 27$ & 1 & 1,524 & 4 & 0 \\
\hline 23 & $0: 13: 18$ & $0: 28: 15$ & $0: 41: 33$ & 1 & 1,674 & 4 & 0 \\
\hline 31 & $0: 12: 50$ & $0: 27: 00$ & $0: 39: 50$ & 1 & 1,899 & 4 & 0 \\
\hline 78 & $0: 12: 20$ & $0: 35: 43$ & $0: 48: 03$ & 2 & 2,350 & 4 & 2 \\
\hline 201 & $0: 12: 37$ & $1: 14: 39$ & $1: 27: 16$ & 3 & 3,269 & 4 & 3 \\
\hline
\end{tabular}

Figure 10. Synthesis of the results with 1Generalize.

\section{Conclusions and Future Work}

The use of regular rectangular grid as a partitioning method seems to be the most timeefficient, whatever the type of entity treated (polylines or polygons). The contextual method produces a better simplification, as the partitioning grid does not interfere with the result. However, it comes at a cost, as the processing time is higher. Splitting the lines according to a rectangular grid, even though it represents the quickest method, creates too many fixed points and affects the quality of the simplified polylines. The aesthetic aspect makes this method less interesting than the contextual one. This is the main reason why this solution won't be tested further and why the future studies should focus on contextual strategies.

Concerning the partitioning using administrative or watershed areas, the idea for a division taking the type of entity into consideration is interesting and needs to be investigated further. For now, the main problem impacting the time-efficiency is the geometry of each zone that can be very complex, which slows down spatial queries; 
perhaps a simplification of watersheds as well as administrative areas could be a way to solve it. Then, more tests need to be run to see if this method proves to be quicker than the contextual one with a minor loss of quality. More generally, we plan to conduct much more experiments, with other partitioning and reconciliation methods, with generalization processes that require more context than the simplification algorithms, and with more robust distribution architectures.

For now, we only carried out experiments with the processing of a single algorithm on a single layer of the map, but a more realistic generalization processes needs to handle all the map layers and orchestrate the application of a large number of algorithms (Regnauld et al. 2014). If the assumption that the optimal distribution strategy depends on the feature type and the amount of required context is true, a complete generalization process would require multiple distribution strategies, which might not be a feasible solution. In order to step up, and really make map generalization scalable, we have to develop global distribution models, as 1 Generalize does, or maybe include the distribution issue into the generalization orchestration models. Finally, generalization will more and more have to process big data, e.g. OpenStreetMap, which is then provided by tiles (Sester et al. 2014), so the techniques to distribute generalization processes will surely be used to deal with this amount of data. The development of vector tiles to render maps on the web (Gaffuri 2012) is another example of the future application of distributed generalization processes, and in both applications, the future work described in this conclusion will be fundamental.

\section{References}

Anders, K.-H. 2003. A hierarchical graph clustering approach to find groups of objects. In: Proceedings of 5th ICA Workshop on Progress in Automated Map Generalization. Paris, France.

Basaraner, M., Selcuk, M. 2008. A structure recognition technique in contextual generalization of buildings and built-up areas. The Cartographic Journal, 45(4), pp.274-285.

Brédif, M., Vallet, B., Ferrand, B. 2015. Distributed dimensionality-based rendering of lidar point clouds. In: International Archives of Photogrammetry Remote Sensing and Spatial Information Sciences (GeoBigData). XL-3/W5, Montpellier, France.

Briat, M.-O., Monnot, J.-L., Punt, E. M. 2011. Scalability of contextual generalization processing using partitioning and parallelisation. In: Proceedings of 14th ICA Workshop on Generalization and Multiple Representation. Paris, France.

Bucher, B., Brasebin, M., Buard, E., Grosso, E., Mustière, S., Perret, J. 2012. GeOxygene: Built on top of the expertness of the french NMA to host and share advanced GI science research results. In: E. Bocher, M. Neteler, eds. Geospatial free and open source software in the 21st century. Berlin Heidelberg: Springer, pp. 21-33.

Bundy, G. L., Jones, C. B., Furse, E. 1995. Holistic generalization of large-scale cartographic data. In: J.-C. Müller, J.-P. Lagrange, R. Weibel, eds. GIS and Generalization: Methodology and Practice. London: Taylor \& Francis, pp. $106-119$.

Burghardt, D., Neun, M. 2006. Automated sequencing of generalization services based on collaborative filtering. In: M. Raubal, H.J. Miller, A.U. Frank, M.F. Goodchild, eds. Geographic Information Science - 4th International Conference GIScience. Münster, Germany: IFGI prints, pp. 41-46.

Chaudhry, O. Z., Mackaness, W. A. 2008. Partitioning techniques to make manageable the generalization of national spatial datasets. In: ICA Workshop on Generalization and Multiple Representation. Montpellier, France.

Chaudhry, O. Z., \& Mackaness, W. A. 2010. DTM generalization: Handling large volumes of data for Multi-Scale mapping. The Cartographic Journal, 47(4), pp.360-370.

Douglas, D. H., Peucker, T. K. 1973. Algorithms for the reduction of the number of points required to represent a digitized line or its caricature. Cartographica: The International Journal for Geographic Information and Geovisualization, 10(2), pp.112-122.

Foerster, T., Stoter, J., Kraak, M.-J. 2010. Challenges for Automated Generalization at European Mapping Agencies: A Qualitative and Quantitative Analysis. The Cartographic Journal, 47(1), pp.41-54.

Gaffuri, J., 2012. Toward web mapping with vector data. In: N. Xiao, M.-P. Kwan, M. Goodchild, S. Shekhar, eds. Geographic Information Science. Berlin Heidelberg: Springer, pp. 87-101.

Lokhat, I., Touya, G. 2016. Enhancing Building Footprints with Squaring Operations. Journal of Spatial Information Science 13, pp.33-60.

Regnauld, N. 2014. 1Generalize: 1Spatial's new automatic generalization platform. In: Proceedings of 17th ICA Workshop on Generalization and Multiple Representation. Vienna, Austria.

Regnauld, N., Touya, G., Gould, N., Foerster, T. 2014. Process modelling, web services and geoprocessing. In: D. Burghardt, C. Duchêne, W. Mackaness, eds. Abstracting Geographic Information in a Data Rich World. Berlin Heidelberg: Springer, pp. 198-225. 
Renard, J., Gaffuri, J., Duchêne, C. 2010. Capitalisation problem in research - example of a new platform for generalization: CartAGen. In: Proceedings of 11th ICA Workshop on Generalization and Multiple Representation. Zurich, Switzerland.

Ruas, A., Plazanet, C. 1996. Strategies for automated generalization. In: 7th International Symposium on Spatial Data Handling. Delft, Netherlands, pp. 319-336.

Sester, M., Jokar Arsanjani, J., Klammer, R., Burghardt, D., Haunert, J.-H., 2014. Integrating and generalizing volunteered geographic information. In: D. Burghardt, C. Duchêne, W. Mackaness, eds. Abstracting Geographic Information in a Data Rich World. Cham, Switzerland: Springer International Publishing, pp. 119-155.

Stanislawski, L. V., Falgout, J., Buttenfield, B. P. 2015. Automated extraction of natural drainage density patterns for the conterminous United States through High-Performance computing. The Cartographic Journal, 52(2), pp.185-192.

Stoter, J., van Smaalen, J., Bakker, N., Hardy, P. 2009. Specifying Map Requirements for Automated Generalization of Topographic Data. The Cartographic Journal, 46(3), pp.214-227.

Thiemann, F., Warneke, H., Sester, M., Lipeck, U. 2011. A scalable approach for generalization of land cover data. In: S. Geertman, W. Reinhardt, F. Toppen, eds. Advancing Geoinformation Science for a Changing World. Berlin, Heidelberg: Springer, pp. 399-420.

Thiemann, F., Werder, S., Globig, T., Sester, M. 2013. Investigations into partitioning of generalization processes in a distributed processing framework. In: M. F. Buchroithner, ed. Proceedings of the 26th International Cartographic Conference. Dresden, Germany.

Touya, G. 2010. Relevant space partitioning for collaborative generalization. In: Proceedings of 12th ICA Workshop on Generalization and Multiple Representation. Zurich, Switzerland.

Visvalingam, M., Wyatt, J. D. 1993. Line generalization by repeated elimination of points. The Cartographic Journal, 30(1), pp.46-51.

Visvalingam, M., Whelan, J. C. 2016. Implications of Weighting Metrics for Line Generalization with Visvalingam's Algorithm, The Cartographic Journal, 53(3), pp.253-267. 